OPEN ACCESS

Edited by:

Holmes Finch,

Ball State University, United States

Reviewed by:

Laszlo Hunyadi,

University of Debrecen, Hungary

Fritz Günther,

University of Milano-Bicocca, Italy

*Correspondence:

Kim F. Nimon

kim.nimon@gmail.com

Specialty section:

This article was submitted to

Quantitative Psychology and

Measurement,

a section of the journal

Frontiers in Psychology

Received: 29 December 2019 Accepted: 15 December 2020

Published: 03 February 2021

Citation:

Nimon KF (2021) MOWDOC: A Dataset of Documents From Taking

the Measure of Work for Building a

Latent Semantic Analysis Space.

Front. Psychol. 11:523494

doi: $10.3389 /$ fpsyg.2020.523494

\section{MOWDOC: A Dataset of Documents From Taking the Measure of Work for Building a Latent Semantic Analysis Space}

\author{
Kim F. Nimon* \\ Human Resource Development, University of Texas at Tyler, Tyler, TX, United States
}

Keywords: latent semantic analysis, semantic survey response theory, surveys, jingle-jangle fallacies, work, organization

\section{INTRODUCTION}

For organizational researchers employing surveys, understanding the semantic link between and among survey items and responses is key. Researchers like Schwarz (1999) have long understood, for example, that item order can impact survey responses. To account for "item wording similarity," researchers may allow item error variances to correlate (cf. Rich et al., 2010, p. 625). Other researchers, such as Newman et al. (2010), have pointed to semantic similarity between items as support for the premise that work engagement is like old wine in a new bottle.

Recently, organizational researchers (e.g., Arnulf et al., 2014, 2018) have been able to use latent semantic analysis (LSA) and semantic survey response theory (SSRT) to quantify the semantic similarity between and among scales, items, and survey responses. Latent semantic analysis is a computational model that assesses similarity in language where the similarity of any "given word (or series of words) is given by the context where this word is usually found" (Arnulf et al., 2020, p. 4). Latent semantic analysis involves establishing a semantic space from a corpus of existing documents (e.g., journal articles, newspaper stories, item sets). The corpus of documents is represented in a word-by-document matrix and then transformed into an LSA space through singular value decomposition. The reduced LSA space can be used to assess the semantic similarity of documents within the space as well as new documents that are projected onto the space.

Patterns of semantic similarity resulting from LSA have accounted for a substantive amount of variability in how individuals respond to survey items that purport to measure (a) transformational leadership, motivation, and self-reported work outcomes (60-86\%; Arnulf et al., 2014), (b) employee engagement and job satisfaction (25-69\%; Nimon et al., 2016), and (c) perceptions of a trainee program, intrinsic motivation, and work outcomes (31-55\%, Arnulf et al., 2019). It also appears that personality, demographics, professional training, and interest in the subject matter have an impact on the degree to which an individual's responses follow a semantically predictable pattern (Arnulf et al., 2018; Arnulf and Larsen, 2020, Arnulf et al., 2020). While being able to objectively access the degree to which survey responses are impacted by semantics is a great step forward in survey research, such research is often conducted with LSA spaces that are not open and therefore not customizable except by those that have access to the body of text upon which the LSA space is built. In this day of open science, researchers need access not only to the LSA space on which semantic survey research may be based but also to the underlying corpus of text to determine whether choices made in the generation of the LSA space have an impact on the results found.

Researchers may not be able to create their own LSA spaces for a number of reasons, including the fact that on some occasions it is difficult to collect a representative corpus of text (Quesada, 2011). However, building an LSA space allows researchers to customize the space including the 
application of weighting schemes and the level of dimensionality for the LSA space. As shown by Arnulf et al. (2018), the dimensionality of the LSA space is a factor when using an LSA space to predict empirical correlations from scale item cosines. To help address the barrier to creating an LSA space for use in the analysis of scale items in organizational research, this report provides a dataset of documents from measures reviewed in Taking the Measure of Work. In Taking the Measure of Work, Fields provided the items for 324 scales and subscales which cover the areas of job satisfaction, organizational commitment, job characteristics, job stress, job roles, organizational justice, work-family conflict, person-organization fit, work behaviors, and work values. The MOWDOC dataset presented in this manuscript provides the documents necessary to create a semantic space from the item sets presented in Fields's Taking the Measure of Work.

\section{MOWDOC}

The dataset presented in this manuscript can be accessed via https://doi.org/10.6084/m9.figshare.13298165. The dataset contains five variables for each of the 324 scales and subscales in Fields (2002). The variable ScaleName identifies the name of the measure as reported in Fields as well as subscale(s) as appropriate, where subscale names are preceded by a colon. The variable ScaleRef identifies the reference from which Fields obtained the items.

The variable ScaleID is a unique identifier for each scale/subscale. The first two characters of ScaleID identify the type of measure as delineated by Fields (2002), where JS denotes job satisfaction, OC organizational commitment, JC job characteristics, JT job stress, JR job roles, OJ organizational justice, WC work-family conflict, PO person organization fit, WB work behaviors, and WV work values. The next three characters identify the page number on which the item set first appears in Fields. The remaining characters denote subscale(s) as appropriate.

The variable ScaleDoc contains the document text for each scale/subscale. The scale documents were created as follows. Item texts and associated metadata from Fields (2002) were manually entered into a comma delimited file and verified by an independent and separate individual. To create the variable ScaleDoc, an R script was used to create a character vector by merging all item texts for a given scale/subscale where measures containing multiple item sets or subscales were treated as separate documents. The character vector was tokenized using the tokens function from the quanteda package (Benoit et al., 2018), which also removed all characters in the Unicode "Punctuation" $[\mathrm{P}]$ class. The tokens were then sorted so as to not violate the copyright of the scale publishers. Finally, the tokens were merged into a single character vector.

The variable ScaleSize identifies the number of words for each measure that ranges from 3 to 563 . The hedonism subscale from the Work Values Survey (Schwartz, 1994) has the fewest with two items and the Inventory of Stressful Events (Motowidlo et al., 1986) has the largest with 45 items. The mean number of words across all scales is 67 with an SD of 60 . Across all 324 documents, there are a total of 21,741 words.

\section{EXAMPLE USAGE}

The $\mathrm{R}$ code that demonstrates how the MOWDOC dataset can be used to create an LSA space and fold a new scale ${ }^{1}$ into the created LSA space can be accessed at https://doi.org/10.6084/m9. figshare.13298177. In general, the code follows the example in Wild (2007) and the Wild (2015) demonstration of the famous Landauer et al. (1998) example. Document-feature matrices were created using the $d f m$ function from the quanteda package (Benoit et al., 2018), rather than using the textmatrix function in the lsa package (Wild, 2015). Amongst other differences, the $d f m$ function optimally creates a sparse matrix of documents and features.

Here is the $\mathrm{R}$ code following a typical LSA process:

First, a text matrix was constructed using the input text. In the demonstration provided, five different document-feature matrices and associated word clouds were created to illustrate the nuances associated with stemming words and removing stop words.

Second, an LSA space with full dimensionality was created and used to verify that the document-feature matrix could be reproduced.

Third, an LSA space with reduced dimensionality was created.

Fourth, document-to-document correlations and cosines were computed using the original document-feature matrix and the reduced LSA space.

Fifth, a new document was folded into the reduced LSA space.

Sixth, correlations and cosines with the new document were created.

\section{STRENGTHS AND LIMITATIONS}

The MOWDOC datasets contains the item texts for the scales/subscales in the book of Taking the Measure of Work. With this dataset, researchers can customize their LSA spaces to fit their research interests including the consideration of stop words, word stemming, and weighting schemes. Note, for example, the differences in the word clouds represented in Figure 1 that result when the MOWDOC dataset was used to generate a document-feature matrix with different parameters. Not only did each document-feature matrix contain a different number of features ${ }^{2}$, the word most frequently used across multiple scales

\footnotetext{
${ }^{1}$ The file JS.csv contains the items for the Hackman and Oldham (1980) job satisfaction scale and can be accessed at https://doi.org/10.6084/m9.figshare. 13298168

${ }^{2}$ The document-feature matrix with no stemming or removal of stop words yielded 2,564 features ( 7.9 features on average per scale) and was $98.5 \%$ sparse. The document-feature matrix with no stemming and English stop words from the lsa package (Wild, 2015) removed yielded 2,253 features (7.0 features on average per scale) and was $99.2 \%$ sparse. The document-term matrix with no stemming and English stop words from the quanteda package (Benoit et al., 2018) removed yielded 2,433 features ( 7.5 features on average per scale) and was $99.0 \%$ sparse. The document-term matrix with stemming and English stop words from the quanteda package (Benoit et al., 2018) removed yielded 1,704 features (5.3 features on average per scale) and was $98.6 \%$ sparse.
} 


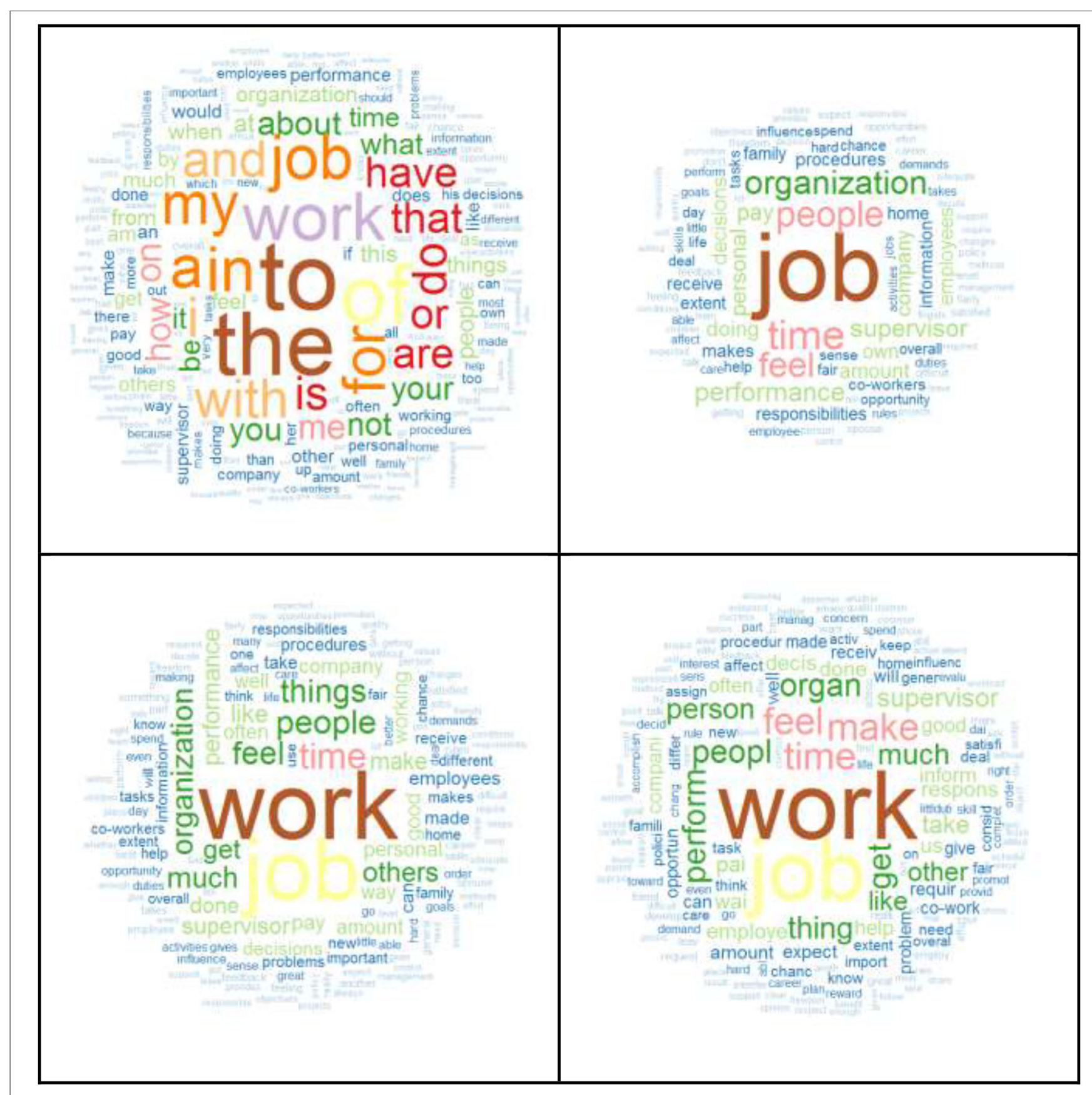

FIGURE 1 | Word clouds on MOWDOC document-feature matrices. Upper-left figure based on matrix with no stemming or stop words removed. Upper-right figure based on matrix with no stemming and English stop words from the Isa package (Wild, 2015) removed. Lower-left figure based on matrix with no stemming and English stop words from the quanteda package (Benoit et al., 2018) removed. Lower-right figure based on matrix with stemming and English stop words from the quanteda package (Benoit et al., 2018) removed.

differed according to how the document texts were "sanitized" (cf. Wild, 2007). In the matrix that hasn't been sanitized with no stemming or removal of stop words, "to" occurs in $76 \%$ (247) of the scales/subscales. When the matrix has no stemming but English stop words from the lsa package (Wild, 2015) are removed, "job" occurred in 48\% (157) of the scales/subscales.
A matrix with no stemming although English stop words from the quanteda package (Benoit et al., 2018) have been removed, "work" occurred in 53\% (172) of the scales/subscales. With stemming and English stop words from the quanteda package (Benoit et al., 2018) removed, "work" occurred in 56\% (181) of the scales/subscales. While it should not come as a surprise that 
"work" is the predominant word used across scales published in a book that considers the "Measure of Work," it could be considered problematic to create an LSA space where such a relevant word was removed.

While making the document texts upon which to build an LSA space available is a strength, it might also be a limitation as resulting LSA spaces may yield over-fitted solutions when researchers assess the semantic similarity of item sets (cf. Larsen et al., 2008). It might also be a limitation that the documentfeature matrices from the MOWDOC dataset tend to be sparse. Across the different "sanitization" schemes previously outlined, all matrices were at least $98.4 \%$ sparse. The dataset is also limited in that it did not preserve the word order of the original item sets. As a reviewer noted, this limits the use of the dataset to document-based models like LSA. In addition, the dataset is small for a source corpus for LSA. With 324 documents and 2,564 unique words, the use of the MOWDOC dataset may be limited beyond the example usage presented.

Clearly more research is needed to determine how the MOWDOC dataset can validly be used to inform survey research. However, even with the stated limitations, the MOWDOC dataset appears to be useful. Take for example the lsaCos.csv file that results from running the demonstration code located at https://doi.org/10.6084/m9.figshare.13298177. It yields the cosines between scales/subscales from the LSA space that was built using a document-feature matrix that was stemmed and void of English stop words contained

\section{REFERENCES}

Arnulf, J. K., Dysvik, A., and Larsen, K. (2019). Measuring semantic components in training and motivation: a methodological introduction to the semantic theory of survey response. Hum. Resour. Develop. Q. 30, 17-38. doi: 10.1002/hrdq.21324

Arnulf, J. K., and Larsen, K. R. (2020). Culture blind leadership research: how semantically determined survey data may fail to detect cultural differences. Front. Psychol. 11:176. doi: 10.3389/fpsyg.2020.00176

Arnulf, J. K., Larsen, K. R., and Martinsen, Ø. L. (2018). Semantic algorithms can detect how media language shapes survey responses in organizational behaviour. PLoS ONE 13:e0207643. doi: 10.10371/journal.pone.0207643

Arnulf, J. K., Larsen, K. R., Martinsen, Ø. L., and Bong, C. H. (2014). Predicting survey responses: how and why semantics shape survey statistics on organizational behaviour. PLOS ONE 9:e106361. doi: 10.10371/journal.pone.0106361

Arnulf, J. K., Nimon, K., Larsen, K. R., Hovland, C. V., and Arnesen, M. (2020). The priest, the sex worker, and the CEO: measuring motivation by job type. Front. Psychol. 11:1321. doi: 10.3389/fpsyg.2020.01321

Benoit, K., Watanabe, K., Wang, H., Nulty, P., Obeng, A., Müller, S., et al. (2018). quanteda: An R package for the quantitative analysis of textual data. J. Open Sour. Softw. 3:774. doi: 10.21105/joss.00774

Cook, J. D., Hepworth, S. J., Wall, T. D., and Warr, P. B. (1981). The Experience of Work: A Compendium of 249 Measures and Their Use. London: Academic Press.

Fields, D. L. (2002). Taking the Measure of Work: A Guide to Validated Scales for Organizational Research and Diagnosis. Thousand Oaks, CA: Sage Publications. doi: 10.4135/9781452231143

Hackman, J. R., and Oldham, G. R. (1980). Work Redesign. Reading: AddisonWesley.

Hersen, M., and Thomas, J. C. (Eds.). (2003). Comprehensive Handbook of Psychological Assessment, Volume 4: Industrial/Organizational Assessment. New York, NY: Wiley. in the quanteda package (Benoit et al., 2018). Notably, the cosine between the OCBO item set Williams and Anderson (1991, WB241B) and the generalized compliance item set from Smith et al. (1983, WB245B) is 0.92. Interestingly, the cosine reflects the fact that some of the items representing $O C B O$, including "attendance at work is above the norm" and "great deal of time spent with personal phone conversation," were selected from the Smith et al. (1983) generalized compliance scale.

Researchers might also fold additional items sets onto the LSA space built from Taking the Measure of Work to assess their semantic similarity with item sets presented in Fields (2002). For example, folding the Hackman and Oldham (1980) job satisfaction item set into the LSA space previously described yields a high cosine (0.86) with the general satisfaction item set from Jackman and Oldham (1974). Future work could include adding item texts from other compendiums of organizational research scales including those of Cook et al. (1981), Price and Mueller (1986), and Hersen and Thomas (2003), as well as submitting the existing dataset to the Semantic Scale Network offered by Rosenbusch et al. (2020).

\section{AUTHOR CONTRIBUTIONS}

The author confirms being the sole contribution of this work and has approved it for publication.

Jackman, J. R., and Oldham, G. R. (1974). The job diagnostic survey: an instrument for the diagnosis of jobs and the evaluation of job redesign project. Tech. Rep. No. 4. New Haven, CT: Yale University, Department of Administrative Sciences.

Landauer, T. K., Foltz, P. W., and Laham, D. (1998). An introduction to latent semantic analysis. Discourse Proces. 25, 259-284. doi: 10.1080/016385380954028

Larsen, K., Nevo, D., and Rich, E. (2008). "Exploring the semantic validity of questionnaire scales," in Proceedings of the 41st annual Hawaii International Conference on System Sciences (Waikoloa, HI), 1-10.

Motowidlo, S. J., Packard, J. S., and Manning, M. R. (1986). Occupational stress: its causes and consequences for job performance. J. Appl. Psychol. 71, 618-629. doi: 10.1037/0021-9010.71.4.618

Newman, D. A., Joseph, D. L., and Hulin, C. L. (2010). "Job attitudes and employee engagement: considering the attitude "Afactor," in The Handbook of Employee Engagement: Perspectives, Issues, Research, and Practice, eds S. L. Albrecht (Northampton, MA: Edward Elgar), 43-61.

Nimon, K., Shuck, B., and Zigarmi, D. (2016). Construct overlap between employee engagement and job satisfaction: a function of semantic equivalence? J. Happiness Stud. 17, 1149-1171. doi: 10.1007/s10902-0159636-6

Price, J., and Mueller, C. (1986). Handbook of Organizational Measurement. Marshfield, MA: Pittman.

Quesada, J. (2011). “Creating your own LSA spaces," in Handbook of Latent Semantic Analysis, T. K. Landauer, D. S. McNamara, S. Dennis, and W. Kintsch (New York, NY: Routledge), 71-88.

Rich, B. L., Lepine, J. A., and Crawford, E. R. (2010). Job engagement: antecedents and effects on job performance. Acad. Manag. J. 53, 617-635. doi: 10.5465/amj.2010.514 68988

Rosenbusch, H., Wanders, F., and Pit, I. L. (2020). The semantic scale network: an online tool to detect semantic overlap of psychological scales and 
prevent scale redundancies. Psychol. Methods 25, 380-392. doi: 10.1037/met0 000244

Schwartz, S. H. (1994). Are there universal aspects in the structure and contents of human values? J. Soc. Issues 50, 19-45. doi: 10.1111/j.1540-4650.1994.tb01196.x

Schwarz, N. (1999). Self-reports - how the questions shape the answers. Am. Psychol. 54, 93-105. doi: 10.1037/0003-066X.54.2.93

Smith, C. A., Organ, D. W., and Near, J. P. (1983). Organizational citizenship behavior: its nature and antecedents. J. Appl. Psychol. 68, 653-663. doi: 10.1037/0021-9010.68.4.653

Wild, F. (2007). “An LSA package for R," in Proceedings of the 1st International Conference on Latent Semantic Analysis in Technology Enhanced Learning (LSA-TEL'07) (Heerlen), 11-12.

Wild, F. (2015). lsa: Latent Semantic Analysis (R package version 0.73.1) [Computer software]. Retrieved from: https://CRAN.R-project.org/package= lsa (accessed November 28, 2020).
Williams, L. J., and Anderson, S. E. (1991). Job satisfaction and organizational commitment as predictors of organizational citizenship and inrole behaviors. J. Manag. 17, 601-617. doi: 10.1177/10490639107 00305

Conflict of Interest: The author declares that the research was conducted in the absence of any commercial or financial relationships that could be construed as a potential conflict of interest.

Copyright (C) 2021 Nimon. This is an open-access article distributed under the terms of the Creative Commons Attribution License (CC BY). The use, distribution or reproduction in other forums is permitted, provided the original author $(s)$ and the copyright owner(s) are credited and that the original publication in this journal is cited, in accordance with accepted academic practice. No use, distribution or reproduction is permitted which does not comply with these terms. 